\title{
A Study on Thrombus Formation in Case of Type B Aortic Dissection and Its Hematocrit Dependence
}

\author{
Alireza Jafarinia ${ }^{1 *}$, Thomas Stephan Müller ${ }^{2}$, Ursula Windberger ${ }^{3}$, Günter Brenn ${ }^{2}$, Thomas Hochrainer ${ }^{1}$ \\ ${ }^{1}$ Institute of Strength of Materials/Graz University of Technology \\ Kopernikusgasse 24/I, Graz, Austria \\ alireza.jafarinia@tugraz.at; hochrainer@tugraz.at \\ ${ }^{2}$ Institute of Fluid Mechanics and Heat Transfer/Graz University of Technology \\ Inffeldgasse 25/F, Graz, Austria \\ t.mueller@tugraz.at; guenter.brenn@tugraz.at \\ ${ }^{3}$ Center of Biomedical Research/Medical University of Vienna \\ Währingergürtel 18-20, 1090 Vienna, Austria \\ ursula.windberger@meduniwien.ac.at
}

\begin{abstract}
Aortic dissection is a disease caused by the occurrence of a rupture in the innermost layer of the aortic wall. Due to the pulsation of the heart, blood penetrates through the tear between the layers of the aortic wall, which causes a new, so-called false lumen (FL). The local hemodynamic conditions in the FL significantly contribute to the formation of a thrombus. The level of thrombosis in the FL affects patients' prognosis and chances of survival, in which a complete thrombosis is usually beneficial. In recent studies on platelet deposition in the FL, it is demonstrated that the change in hemodynamic conditions in the FL effectively boosts platelet activation and aggregation in regions of recirculation. Blood coagulation has the highest chance of occurrence in these recirculation regions within the FL. Considering the dominant influence of shear rate in FL thrombosis, the non-Newtonian rheological properties and behaviour of blood play a crucial role. The most important rheological factor is the volume fraction of red blood cells in the blood, i.e., the hematocrit value (HCT), which affects the shear rate dependent viscosity and the yield stress observed in low shear rate flow of blood. In the current work, the influence of the hematocrit value on thrombosis in the FL is investigated. The simulations are done in idealized aortic dissection phantom models employing HCT-dependent non-Newtonian hemodynamics. The values for the HCT were varied within a physiological range. On the one hand, an increase in the total volume of thrombus in time was found for all HCT values. On the other hand, by increasing the HCT values, less thrombus is formed in the FL. This suggests that high HCT values impede thrombus formation due to rheological effects and that patients with higher hematocrit values have less chance of benefiting from complete thrombosis in the FL.
\end{abstract}

Keywords: Aortic Dissection, Thrombus Formation, Hematocrit, Carreau Model

\section{Introduction}

Type B aortic dissection (TBAD) is a severe disease associated with high mortality. TBAD could also lead to complications such as aortic aneurysm, rupture, or malperfusion syndromes [1]. TBAD is initiated by the perforation of the inner layer of the aortic wall, causing its layers to delaminate. As the delamination progresses, a new volume, the so-called FL, is created; hence, the blood flow bifurcates partially from the main blood flow, in the descending part of the aorta (true lumen, TL), into the false lumen. The tear generating the FL propagates due to the pulsation of the blood flow, and eventually, a second tear (re-entry tear) may occur in the descending part of the aorta, merging the flows of both lumens.

The hemodynamic conditions in the FL, including flow disturbance, recirculation, and considerable variability in wall shear stress (WSS), might contribute to the formation and growth of a thrombus [2]. There exist three cases in TBAD false lumen thrombosis, i.e., patent, partial, and complete thrombosis. Tsai et al. [3] showed that partial thrombosis is associated with a higher mortality rate, whereas complete thrombosis of the FL improves patients prognosis [4] and [5]. Therefore, it is important to investigate and understand under which conditions complete thrombosis of the FL occurs.

Thrombus formation is a complex phenomenon comprising many biological and chemical processes. Until now, it is unclear why thrombosis does or does not occur following aortic dissection. Thrombosis, in the case of aortic dissection, is 
dominated by hemodynamic conditions, and it is enhanced in low shear rate regions in the FL [5]. In recent studies on platelet deposition, it is demonstrated that the change in hemodynamic conditions in the false lumen effectively boosts platelet activation and aggregation in regions of recirculation [6]. Blood coagulation has the highest chance of occurrence in these recirculation regions within the FL, where the shear rates are significantly lower than in the TL [7]. Considering the dominant influence of shear rate in false lumen thrombosis, the rheological behaviour of blood plays a crucial role. Blood is a suspension with a non-Newtonian rheological behaviour exhibiting shear thinning and yield stress. Blood rheology mainly depends on the volume fraction of red blood cells in the blood, i.e., the hematocrit value [8], which varies from person to person, ranging between 30 and 55 percent. In the current work, we study the influence of the hematocrit value on thrombosis in the false lumen.

For the thrombus formation simulation, the hemodynamic-based mathematical model developed by Menichini et al. ([2] and [9]) is used. In this model, the thrombus formation is enhanced in low shear rate regions, making it suitable for investigating the influence of HCT variation. To describe the shear-dependent behaviour of blood, the Carreau model is used [10]. The four parameters of the model are obtained by blood rheometry. The rheological model incorporates an additional rheological phenomenon of blood, the tendency of red blood cells to agglomerate and form so-called rouleaux. Those rouleaux need a minimum of shear stress to be broken up; thus, a yield stress is accounted for in the model. The numerical simulations are carried out in the open source software OpenFOAM.

\section{Mathematical modelling of thrombus formation}

The flow in the aortic arch and false lumen is modelled by a modified form of the Navier-Stokes equations,

$$
\rho\left[\frac{\partial \boldsymbol{u}}{\partial t}+(\boldsymbol{u} \cdot \boldsymbol{\nabla}) \boldsymbol{u}\right]=-\boldsymbol{\nabla} p+\boldsymbol{\nabla} \cdot \boldsymbol{\tau}-k_{\mathrm{th}} \phi_{\mathrm{th}} \boldsymbol{u}
$$

where $\tau$ is the Cauchy stress tensor, and the sink term on the right accounts for the dynamic influence of the thrombus on the flow. The quantity $\phi_{\mathrm{th}}\left(c_{\mathrm{BP}}, c_{\mathrm{BP}}\right)=c_{\mathrm{BP}}^{2} /\left(c_{\mathrm{BP}}^{2}+c_{\mathrm{BPt}}^{2}\right)$ indicates local thrombosis as a function of the bounded-platelets concentration $c_{\mathrm{BP}}$, as determined by the thrombus model discussed in the sequel. The model [2] controls the formation of the thrombus based on shear stress, residence time, as well as the concentrations of coagulant and bounded platelets. In this model, thrombus growth is limited or enhanced depending on threshold values for the mentioned quantities (subscript $t$ ). The value of the coefficient $k_{\text {th }}$ in the sink term is large enough to stop the flow where the thrombus is formed [2]. In the current work we may only give a very cursory introduction to the model and refer to [2] for details and to [9] for the employed parameters.

The thrombus formation model consists of the following equations. The model is based on the so-called residence time $T_{\mathrm{R}}$ of liquid components or platelets in the field, which is determined by the transport equation

$$
\frac{\partial T_{\mathrm{R}}}{\partial t}+\boldsymbol{u} \cdot \boldsymbol{\nabla} T_{\mathrm{R}}=D_{T_{\mathrm{R}}} \nabla^{2} T_{\mathrm{R}}+1
$$

High residence time marks areas where the platelets spend more time [2]. The transport equation for the resting and activated platelet concentrations, $c_{\mathrm{RP}}$ and $c_{\mathrm{AP}}$, is [2]

$$
\frac{\partial c_{i}}{\partial t}+\boldsymbol{u} \cdot \boldsymbol{\nabla} c_{i}=D_{\mathrm{P}} \boldsymbol{\nabla}^{2} c_{i}+S_{i}, \quad i=R P, \mathrm{AP} .
$$

Here, $D_{\mathrm{P}}$ denotes the diffusion coefficient of platelets, which is the same for resting and activated platelets. Furthermore, $S_{i}$ denote reaction terms with the so-called coagulant. The coagulant is the lumped effect of all underlying biochemical reactions in the coagulation cascade [2]. In low shear rate areas, there is a production of 
coagulant at the wall based on the conditions specified on the boundary. The diffusion-reaction equation and the wall reaction equation for the coagulant concentration $c$ are,

$$
\begin{aligned}
& \frac{\partial c}{\partial t}=D_{c_{\mathrm{eff}}} \nabla^{2} c+k_{\mathrm{c}} \phi_{\mathrm{th}} \phi_{\dot{\gamma}}, \text { where } \phi_{\dot{\gamma}}={\overline{\dot{\gamma}_{\mathrm{t}}}}^{2} /\left(\overline{\dot{\gamma}}^{2}+\overline{\dot{\gamma}}_{\mathrm{t}}^{2}\right) \text {, and } \\
& \left.D_{c_{\text {eff }}} \frac{\partial c}{\partial n}\right|_{\text {wall }}=\left\{\begin{array}{cl}
k_{c_{\text {wall }}} & \text { if cycle-averaged WSS }<0.2 \mathrm{~Pa} \text { and } c_{\mathrm{BP}} \leq 200 \mathrm{nM}, \\
0 & \text { otherwise, }
\end{array}\right.
\end{aligned}
$$

where $k_{\mathrm{c}}$ and $k_{c_{\mathrm{wall}}}$ are reaction constants [2]. Finally, the rate of production of bounded platelets concentration $c_{\mathrm{BP}}$ is given by

$$
\frac{\partial c_{\mathrm{BP}}}{\partial \mathrm{t}}=k_{\mathrm{BP}} \phi_{\mathrm{BP}} \phi_{\dot{\gamma}} c_{\mathrm{AP}}, \text { where } \phi_{\mathrm{BP}}=\left(c^{2} /\left(c^{2}+c_{\mathrm{t}}^{2}\right)\right)\left(\bar{T}_{\mathrm{R}}^{2} /\left(\bar{T}_{\mathrm{R}}^{2}+T_{\mathrm{R}_{\mathrm{t}}}^{2}\right)\right)
$$

with $\bar{T}_{\mathrm{R}}$ being the cycle-averaged residence time, $T_{\mathrm{R}_{\mathrm{t}}}$ a threshold value and $k_{\mathrm{BP}}$ a reaction constant. Regions with high concentrations of bounded platelets are considered as thrombus, where the blood flow gets inhibited by the modification of the Navier-Stokes equations, Eq. (1).

\section{Rheological modelling}

The rheological model of blood as a shear-thinning liquid with a yield stress determines the extra stress or the rate of deformation tensors as

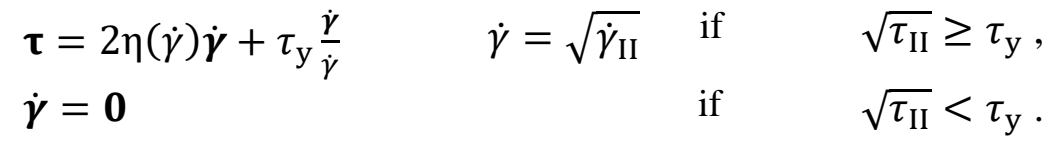

The fluid does not flow if the stress magnitude is below the yield stress. The second invariants of the extra stress and rate of deformation tensors $\boldsymbol{\tau}$ and $\dot{\gamma}$, respectively, are (Cartesian coordinates $\mathrm{x}, \mathrm{y}$ for $\mathrm{i}$ or $\mathrm{j}=1,2$ )

$$
\begin{array}{llll}
\tau_{\mathrm{II}}=\frac{1}{2}\left[I_{\tau}^{2}-I I_{\tau}\right] \quad \text { with } \quad I_{\tau}=\sum_{\mathrm{i}} \tau_{\mathrm{ii}} \quad \text { and } \quad I I_{\tau}=\sum_{\mathrm{i}} \sum_{\mathrm{j}} \tau_{\mathrm{ij}} \tau_{\mathrm{ji}} & \\
\dot{\gamma}_{\mathrm{II}}=\frac{1}{2}\left[I_{\dot{\gamma}}^{2}-I I_{\dot{\gamma}}\right] \quad \text { with } \quad I_{\dot{\gamma}}=\sum_{\mathrm{i}} \dot{\gamma}_{\mathrm{ii}} \quad \text { and } & I I_{\dot{\gamma}}=\sum_{\mathrm{i}} \sum_{\mathrm{j}} \dot{\gamma}_{\mathrm{ij}} \dot{\gamma}_{\mathrm{ji}} \quad \dot{\gamma}_{\mathrm{ij}}=\frac{1}{2}\left(\frac{\partial u_{i}}{\partial x_{j}}+\frac{\partial u_{j}}{\partial x_{i}}\right)
\end{array}
$$

In these equations, the shear-rate dependent viscosity and the yield stress appear as liquid material properties, which are modelled as laid out in the following sections.

\subsection{Shear-rate dependent viscosity}

Shear rheometric data of human blood was measured by a rheometer with a double cylindrical gap geometry for shearrates ranging from $1 s^{-1}$ to $1000 s^{-1}$. The flow curves were obtained for four different hematocrit values (HCT=30\%$60 \%$, with a gradation of 10\%) from five different test persons. The shear-thinning behaviour of blood qualifies the Carreau model

$$
\eta(\dot{\gamma})=\eta_{\infty}+\left(\eta_{0}-\eta_{\infty}\right)\left[1+(\lambda \dot{\gamma})^{2}\right]^{\frac{n-1}{2}}
$$


for representing the flow curves. The four quantities $\eta_{0}, \eta_{\infty}, \lambda$ and $n$ are free model parameters to be determined in fitting the model equation to measurements. The data are shown in Fig. 1. The green curves represent the fits with the Carreau model. For evaluating the viscosity at HCT values intermediate to the experiments, a fit of the model parameters was performed. Thus, all four parameters were available as functions of the HCT value. The pink curves correspond to the viscosities calculated with the parameters from the fit for the corresponding hematocrit, as noted next to the curves. For the Newtonian cases, the viscosity was calculated as corresponding to the Reynolds number Re of the flow.

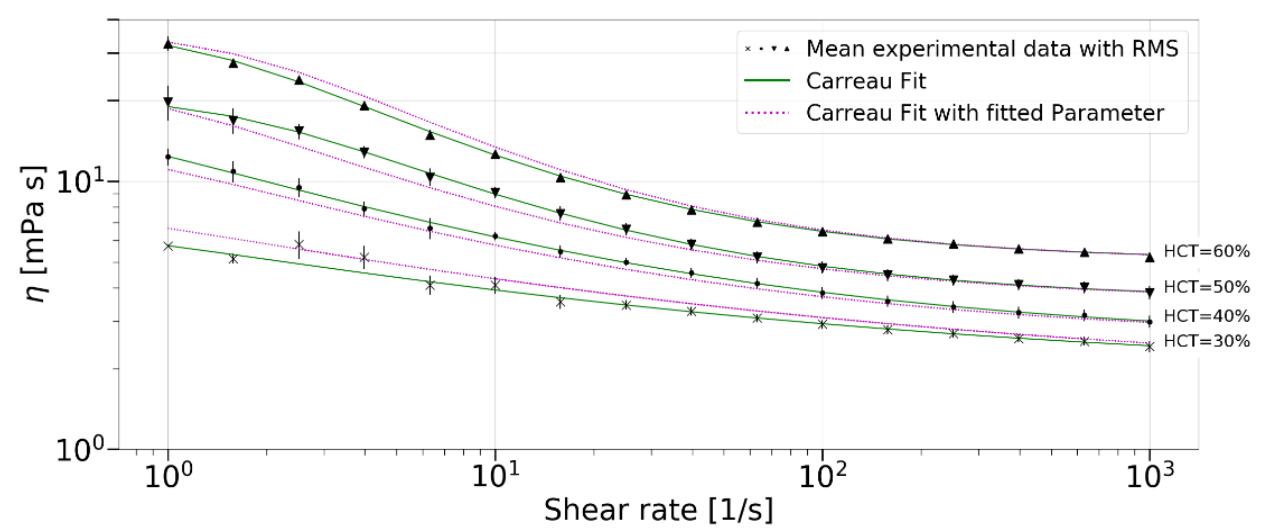

Fig. 1: Shear rheometric data of blood for different hematocrit values. The error bars represent the standard deviations.

\subsection{Yield stress}

The incorporation of a yield stress in the rheological model is based on the mathematical formulation by Merrill [11] as a piece-wise function of the hematocrit value HCT. The model equations

$$
\begin{array}{cc}
\tau_{\mathrm{y}}=7.14 \cdot 10^{-5}(H C T-6.5)^{3} & \text { for } 30 \% \leq \mathrm{HCT} \leq 50 \% \text { and } \\
\tau_{\mathrm{y}}=0.48 \cdot e^{0.044 \cdot H C T} & \text { for HCT }>50 \%
\end{array}
$$

reveal the yield stress in milli-Pascal with the HCT inserted in percent. The model approximation to the experimental data available in the literature is shown in Fig. 2.

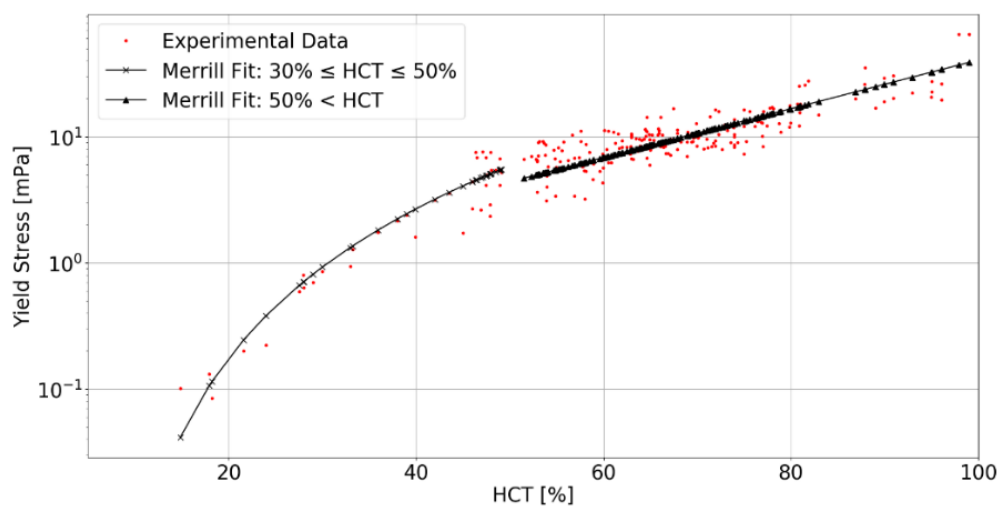

Fig. 2: Experimental and fitted model data for the yield stress depending on the hematocrit value.

\section{Problem formulation}

Thrombus formation in the case of aortic dissection type B was investigated on the 2D geometry of an abstracted aorta given in Fig. 3, with the geometrical parameters in Table 1. For the sake of simplicity, only the ascending aorta, 
aortic arch without carotid arteries, and the descending part of the thoracic aorta were considered. The dissection is realized by splitting up the descending part of the aorta into a TL and an FL, which are connected via a proximal and a distal tear. The ascending part of the aorta has the width $D_{\mathrm{AA}}$ and length $L_{\mathrm{AA}}$. The width of the aortic arch is equal to the one of the ascending part. The radius of curvature is described by $R_{\text {Arc }}$. The arch ends at the angle $\alpha$.



Table 1: Parameters of the geometry. $\alpha=45^{\circ}$

\begin{tabular}{|c|c|}
\hline Parameter & Value $[\mathrm{m}]$ \\
\hline$D_{A A}$ & 0.025 \\
\hline$D_{\mathrm{TL}}$ & 0.0177 \\
\hline$D_{\mathrm{FL}}$ & 0.0182 \\
\hline$H_{\mathrm{T} 1}$ & 0.0091 \\
\hline$H_{\mathrm{T} 2}$ & 0.0091 \\
\hline$L_{\mathrm{AA}}$ & 0.18 \\
\hline$L_{1}$ & 0.0273 \\
\hline$L_{2}$ & 0.0173 \\
\hline$L_{3}$ & 0.0091 \\
\hline$R_{\mathrm{Arc}}$ & 0.03 \\
\hline$B$ & 0.001 \\
\hline
\end{tabular}

\section{Computational fluid dynamics}

The model equations were solved with the finite volume method using the open-source software OpenFOAM. The 2D geometry of the flow domain was discretized into 16000 hexahedral cells, with a refining grading towards the wall to resolve correctly the gradients on the wall surface. A grid independency study was performed to ensure that a refinement of the spatial discretization would not change the outcome of the value $\bar{\phi}_{\mathrm{th}}$. For the numerical schemes, a second-order implicit scheme for the time was chosen, gradient schemes with a second-order central difference and divergence schemes were calculated with a Gaussian first or second-order scheme. The non-orthogonality of wall-normal gradients was taken into account by using an appropriate correction scheme. Laplacian terms are calculated linearly limited by a value of unity. For each simulation, first, the periodic solution is reached, and then the thrombus formation equations are included. For the inlet boundary condition of the velocity, a parabolic profile taken from [12] was imposed, with the no-slip condition for walls. The details about the boundary conditions for the thrombus formation equations can be found in [2] and [9].

\section{Results}

The formation of a thrombus is indicated by the parameter $\phi_{\mathrm{th}}\left(c_{\mathrm{BP}}, c_{\mathrm{BP}_{\mathrm{t}}}\right)$ as introduced in section 2 . Complete thrombosis at a point is indicated if $\phi_{\mathrm{th}}$ approaches the value 1 . Though the value 1 may not be reached, this parameter shows a clear bimodal distribution of values, which are effectively either zero or larger than 0.98 , confer Fig. 8. Note though that the concentration of bounded platelets affects the flow whenever $c_{\mathrm{BP}}>0$. A measure of the volume fraction of thrombosis of the simulation volume $V$ is defined through

$$
\bar{\phi}_{\mathrm{th}}(t)=\frac{1}{V} \int_{\mathrm{V}} \phi_{\mathrm{th}}(x, y, z, t) d V
$$

The rate of thrombosis, subsequently also referred to as the thrombus growth rate, is defined as

$$
\dot{\bar{\phi}}_{\mathrm{th}}(t)=\frac{d}{d t}\left(\bar{\phi}_{\mathrm{th}}(t)\right)=\frac{1}{V} \frac{d}{d t} \int_{\mathrm{V}} \phi_{\mathrm{th}}(x, y, z, t) d V .
$$


In Fig. 4, the volume fraction of thrombosis is shown as a function of time. For all HCT values, the same trend seen in time, with an increase in the amount of formed thrombus. Furthermore, by increasing the HCT values, less thrombus is formed in the FL. In the early stages of thrombus formation up to approximately 10s simulation time, the difference in relative thrombosis for different HCT values is negligible. As time progresses, the difference of the values increases.

The rate of thrombosis is shown in Fig. 5 as a function of time. For all the simulations, the rate of thrombosis hits a peak and drops down to a mostly constant value afterwards. Although the growth rate indicates an oscillatory behaviour, overall, the higher the HCT values, the lower the average growth rate (Fig. 7). Changes in $\bar{\phi}_{\text {th }}(t)$ with HCT for five different times are depicted in Fig. 6. In the early stages of thrombus formation, until about 10s of simulation time, the changes are negligible, and HCT has not played a role yet. With increasing simulation time, an anti-proportional correlation becomes visible between $\bar{\phi}_{\mathrm{th}}(t)$ and HCT.

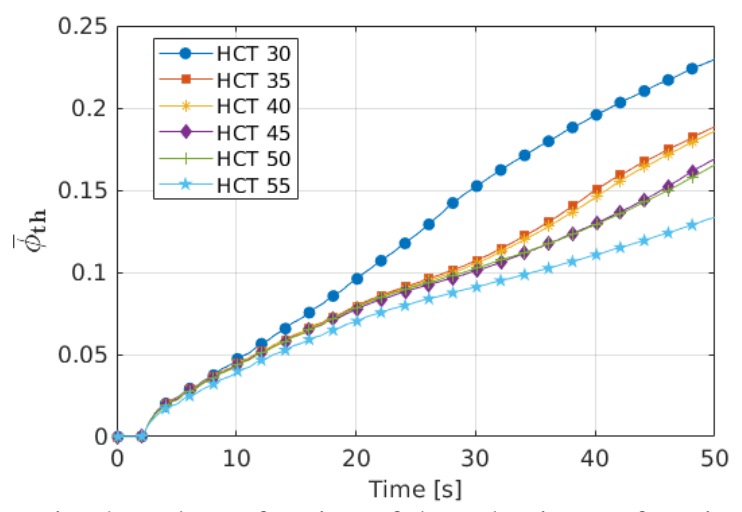

Fig. 4: Volume fraction of thrombosis as a function of time.

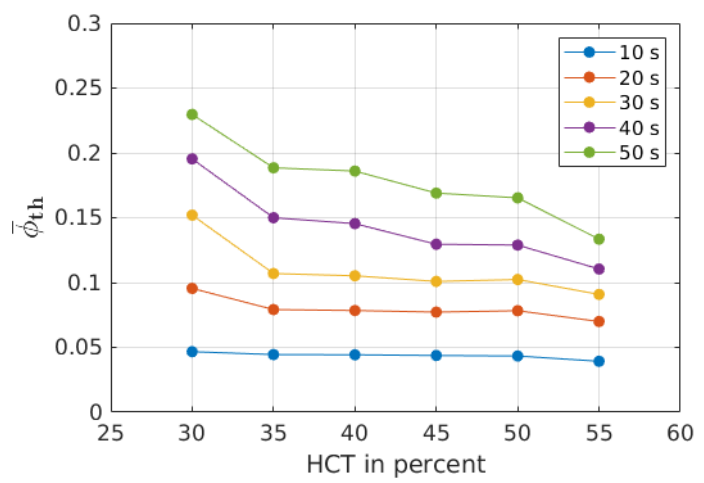

Fig. 6: Volume fraction of thrombosis vs. HCT values.

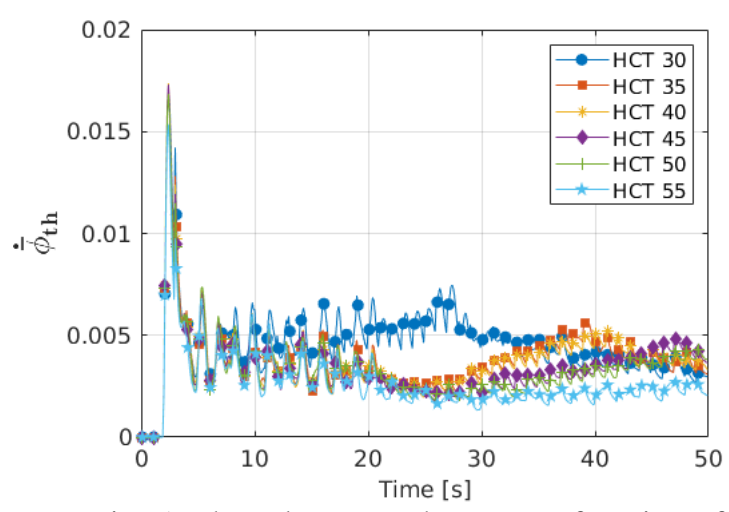

Fig. 5: Thrombus growth rate as a function of time.

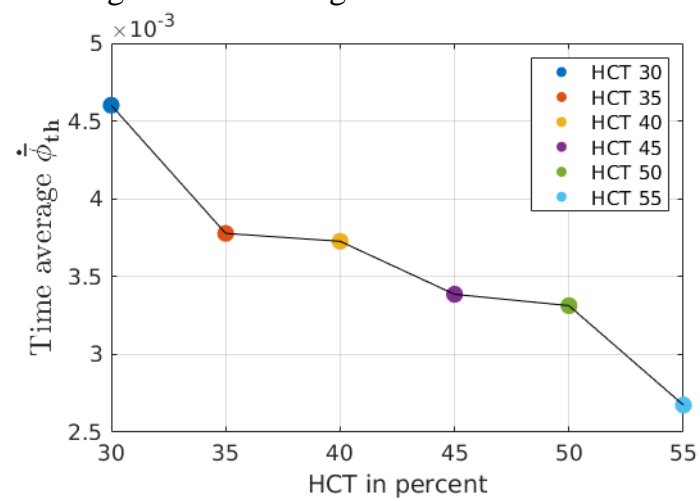

Fig. 7: Average thrombus growth rate vs. HCT values.

Fig. 8 illustrates the FL thrombosis after 50 cardiac cycles. The volume of thrombus in the false lumen decreases with the HCT values. The reason for an increase of HCT values reducing thrombosis likelihood in the false lumen is that:

1. Both, viscosity of blood and shear rate in the simulations has been consistently experiencing growth with the HCT value. High shear rates limit the growth of thrombus.

2. As a result of an increase in viscosity and cycle-averaged shear rate, the cycle-averaged wall shear stress also grows (Fig. 9), which restricts the formation of thrombus by reducing the coagulant production from the wall. The cycleaveraged threshold for the wall shear stress, to cause a flux of coagulant towards the wall and consequently thrombus formation, is $0.2 \mathrm{~Pa}$, this means that for cycle averaged WSS of above $0.2 \mathrm{~Pa}$ thrombus formation will not occur. 
3. Non-Newtonian behaviour of blood causes the velocity profile to be flattened in the vicinity of the false lumen center line, causing steep velocity gradients near the wall, which increases the shear rate near the wall and thus the wall shear stress. The higher the viscosity, the more flattened is the velocity profile. The kinematic viscosity for different HCT values is shown at peak systole in Fig. 10. In recirculation areas with low convection and low shear rate, the viscosity increases with the HCT value. This means at the top of the false lumen, and above the proximal tear, as well as at the bottom of the false lumen and below the re-entry tear, the blood flow experiences more resistance, which forces the flow to go through the areas with lower resistance, thus leading to enhanced shear rates in the FL.

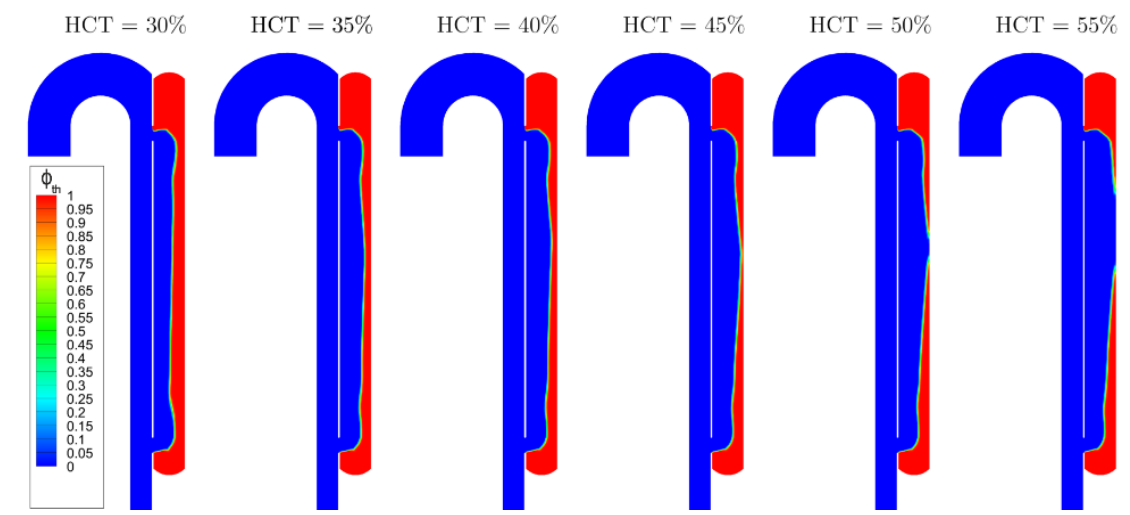

Fig. 8: False lumen thrombosis as indicated by $\phi_{\text {th }}$ for different HTC values after 50 cardiac cycles simulation time.



Fig. 9: Cycle-averaged shear stress before thrombus formation initiation. The cycle-averaged WSS threshold is 0.2 Pa. 


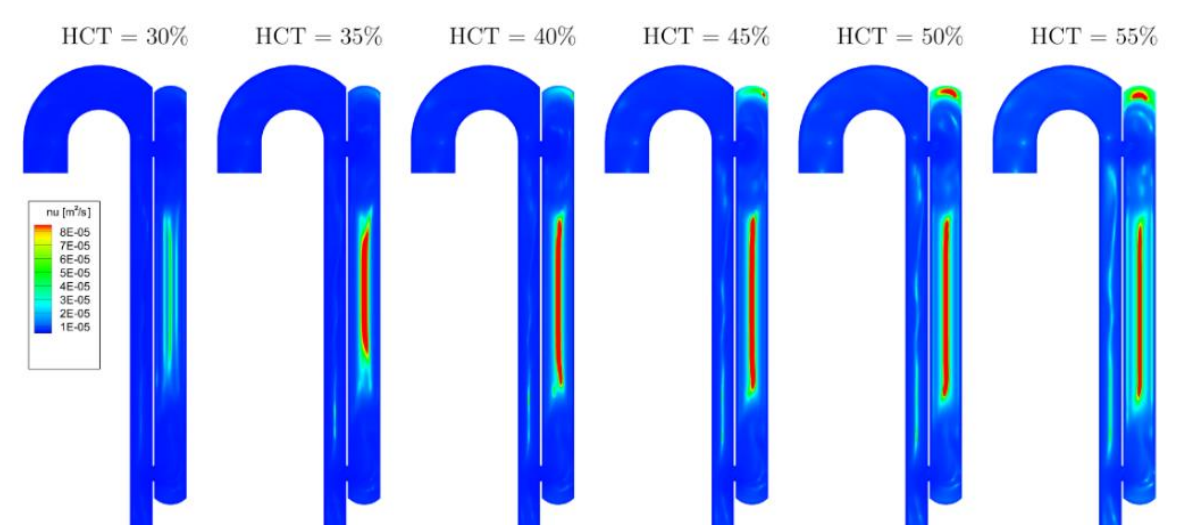

Fig. 10: Kinematic viscosity at 1.1s at peak systole, before thrombus formation.

\section{Conclusion}

This paper presents the application of a hemodynamic-based thrombus formation model for studying the hematocrit dependency of FL thrombosis in TBAD. A rheological model accounting for shear-thinning and a yield stress is applied for the blood flow simulations. Shear rheometric data of human blood was measured by a rheometer. The yield stress was modelled based on data from the literature. Our study predicted a higher probability of complete thrombosis for cases with lower hematocrit values. This is due to an increase in wall shear stress, viscosity and shear rate in the false lumen with the HCT values. The results from the study suggest that patients with higher HCT values have less chance of benefiting from thrombosis in the false lumen, or that they need more time to develop the same state of thrombosis.

\section{Acknowledgements}

The authors gratefully acknowledge funding by TU Graz within the LEAD project "Mechanics, Modelling, and Simulation of Aortic Dissection." Blood rheometry was carried out at the Center of Biomedical Research of the Medical University of Vienna.

\section{References}

[1] C. A. Nienaber, R. E. Clough, N. Sakalihasan, T. Suzuki, R. Gibbs, F. Mussa, M. P. Jenkins, M. M. Thompson, A. Evangelista, J. S. Yeh, N. Cheshire, U. Rosendahl and J. Pepper, "Aortic dissection," Nat. Rev. Dis. Prim., vol. 2, 17 pp., 2016, doi: 10.1038/nrdp.2016.53.

[2] C. Menichini and X. Y. Xu, "Mathematical modeling of thrombus formation in idealized models of aortic dissection: initial findings and potential applications," J. Math. Biol., vol. 73, no. 5, pp. 1205-1226, 2016, doi: 10.1007/s00285-016-0986-4.

[3] T. T. Tsai, A. Evangelista, C. A. Nienaber, T. Myrmel, G. Meinhardt, J. V. Cooper, D. E. Smith, T. Suzuki, R. Fattori, A. Llovet, J. Froehlich, S. Hutchison, A. Distante, T. Sundt, J. Beckman, J. L. Januzzi, Jr., E. M. Isselbacher, and K. A. Eagle, "Partial thrombosis of the false lumen in patients with acute type B aortic dissection," N. Engl. J. Med., vol. 357, no. 4, pp. 349-359, doi: 10.1056/NEJMoa063232.

[4] S. Trimarchi, J. L. Tolennar, F. H. W. Jonker, B. Murray, T. T. Tsai, K. A. Eagle, V. Rampoldi, H. J. M. Verhagen, J. A. van Herwaarden, F. L. Moll, B. E. Muhs and J. A. Elenfteriades, "Importance of false lumen thrombosis in type B aortic dissection prognosis," J. Thorac. Cardiovasc. Surg., vol. 145, no. 3 SUPPL., pp. S208-S212, 2013, doi: $10.1016 / j$.jtcvs.2012.11.048.

[5] M. Anand, K. Rajagopal, and K. R. Rajagopal, "A model incorporating some of the mechanical and biochemical factors underlying clot formation and dissolution in flowing blood," J. Theor. Med., vol. 5, no. 3-4, pp. 183-218, 2003, doi: 10.1080/10273660412331317415. 
[6] J. J. Hathcock, "Flow effects on coagulation and thrombosis.," Arterioscler. Thromb. Vasc. Biol., vol. 26, no. 8, pp. 1729-37, Aug. 2006, doi: 10.1161/01.ATV.0000229658.76797.30.

[7] C. G. Caro, T. J. Pedley, R. C. Schroter, W. A. Seed, and K. H. Parker, The Mechanics of the Circulation. Cambridge: Cambridge University Press, 2011.

[8] A. L. Fogelson and K. B. Neeves, "Fluid Mechanics of Blood Clot Formation," Annu. Rev. Fluid Mech., vol. 47, no. 1, pp. 377-403, 2015, doi: 10.1146/annurev-fluid-010814-014513.

[9] C. Menichini, Z. Cheng, R. G. J. Gibbs, and X. Y. Xu, "Predicting false lumen thrombosis in patient-specific models of aortic dissection," J. R. Soc. Interface, vol. 13, no. 124, 11 pp., 2016, doi: 10.1098/rsif.2016.0759.

[10] P. J. Carreau, "Rheological Equations from Molecular Network Theories," Trans. Soc. Rheol., vol. 16, no. 1, pp. 99-127, 1972, doi: 10.1122/1.549276.

[11] W. Merrill, "Rheology of Blood,” Physiological Review, vol. 49, no. 4, pp. 863-888, 1969.

[12] J. Alastruey, N. Xiao, H. Fok, T. Schaeffter, and C. A. Figueroa, "On the impact of modelling assumptions in multiscale, subject-specific models of aortic haemodynamics," J. R. Soc. Interface, vol. 13, no. 119, 17 pp., 2016, doi: 10.1098/rsif.2016.0073. 